\title{
A Type of Two-Photon Microfabrication System and Experimentations
}

\author{
Peng Wei, ${ }^{1}$ Ning Li, ${ }^{2}$ and Lishuang Feng ${ }^{1}$ \\ ${ }^{1}$ Department of Opto-Eletronics, School of Instrument Science and Opto-Electronics, Beijing University of Aeronautics and \\ Astronautics, Beijing 100083, China \\ ${ }^{2}$ School of Automation Science and Electrical Engineering, Beijing University of Aeronautics and Astronautics, Beijing 100083, China
}

Correspondence should be addressed to Peng Wei, weipeng100@vip.sina.com

Received 5 December 2010; Accepted 29 December 2010

Academic Editors: W. Dai and A. Tounsi

Copyright (c) 2011 Peng Wei et al. This is an open access article distributed under the Creative Commons Attribution License, which permits unrestricted use, distribution, and reproduction in any medium, provided the original work is properly cited.

\begin{abstract}
After the femtosecond laser was invented, two-photon microfabrication technology has been recognized as an important method to fabricate the nanostructure and microstructure. In this paper, the two-photon microfabrication system is described, and some experiments are done. From the experiment results, it can be seen that the resolution of the two-photon microfabrication system can be improved by the expose time, the laser power, and the diffractive superresolution element (DSE). Finally, some threedimensional (3D) microstructure models are fabricated to show the potential of the two-photon microfabrication method.
\end{abstract}

\section{Introduction}

Fabricating smaller structure is one of the goals of the microfabrication technology [1-4]. During the past 10 years, twophoton-induced photopolymerization has been studied in many areas including photonic crystals [5], biochannels, high-resolution AFM tips, 3D optical memory [6], and 3D stamp for imprint [7-9].

At present, the most popular two-photon 3D microfabrication method focuses on the polymerization of photosensitive resin. Compared to the single-photon microfabrication methodology [10], one of the greatest advantages of the twophoton $3 \mathrm{D}$ microfabrication methodology is the true $3 \mathrm{D}$ fabrication ability.

Figure 1 shows the difference between single-photon microfabrication and two-photon microfabrication. In Figure 1, "a" is the photopolymerization point for the single-photon methodology, and " $\mathrm{b}$ " is the photopolymerization point for the two-photon methodology. Obviously, the single-photon methodology photopolymerization can happen only on the surface of the photosensitive resin, but the two-photon methodology photopolymerization can happen both inside and on the surface of the photosensitive resin.

In this paper, we provide a type of two-photon microfabrication system and some experimentation. The whole system and the experimentation could be used to illustrate the potential of the two-photon microfabrication method.

\section{Superresolution Analysis of the Two-Photon Microfabrication}

The free-radical photopolymer is composed of photoinitiators, photosensitizers, and monomers. Photopolymerization is usually concerned with the creation of a polymer through a chain reaction initiated by laser. And the chain of reaction consists of three basic processes: a radical reaction, a monomer radical reaction, and polymerization termination. Based on the principle of two-photon absorption, an initiator is excited from the ground to the excited states by absorbing two photons simultaneously, at the same time the single photon absorption is very weak. After the free-radical photopolymer is produced, the polymerization depends on the concentration of the radicals and the efficiency of the polymerization reaction [11-13].

Under certain conditions, the polymerization process will take place. In this process, the concentration of the radical photopolymer $\xi$ reaches the polymerization critical value $\xi$ th. But nothing will happen if $\xi<\xi$ th. For certain kind of photosensitive resin, the polymerization critical value 


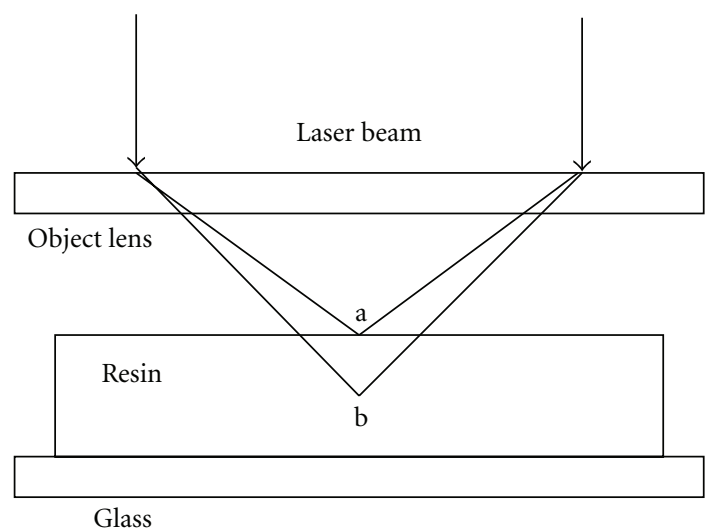

Figure 1: Comparison between single-photon and two-photon methods of microfabrication.

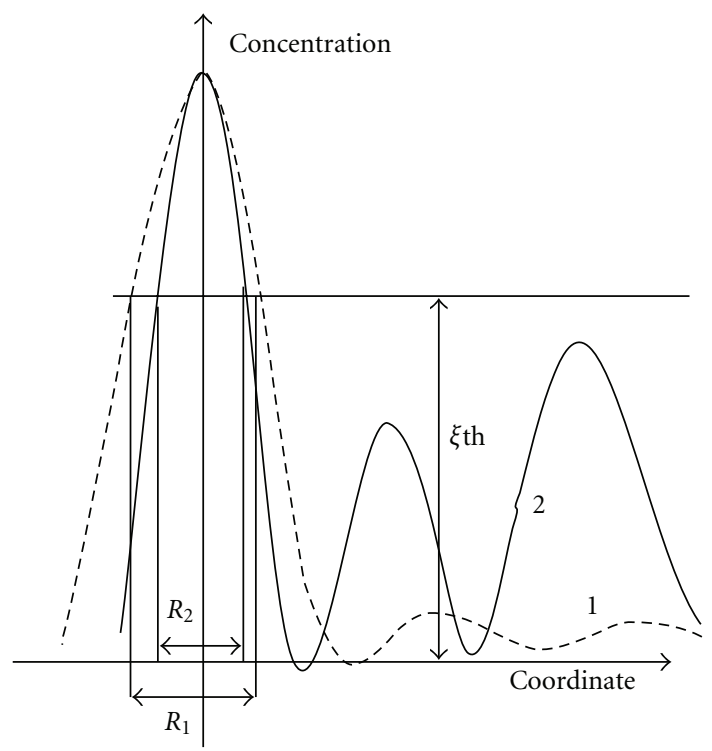

Figure 2: Principle of DSE and superresolution of two-photon polymerization.

is constant. And under certain conditions, the ratio between the intensity of the laser $I$ and the concentration of the radical photopolymer $\xi$ is definite. So the intensity critical value Ith could be used to define the critical value of the two-photon microfabrication, just as the $\xi$ th did. So under certain conditions, the polymerization will take place where the intensity of the laser $I \geq I$ th, but nothing will happen if $I<$ Ith [14].

To reach the superresolution goal of the two-photon polymerization system, the DSE [15-17] is introduced in the system. The DSE is aimed to sharpen the concentration of the radical photopolymer $\xi(t)$; [18-20] its principle is shown in Figure 2.

In Figure 2, the dashed curve 1 is the shape of the concentration of the radical photopolymer without the DSE. And the real curve 2 is the concentration after the DSE is introduced in the system. Based on the critical value $\xi$ th, the polymerization process only happens in the $R_{2}$ area by using the DSE. While without the DSE, the polymerization process happens in the $R_{1}$ area. Obviously, the area of $R_{2}$ is smaller than $R_{1}$. So after the DSE is introduced in the system, the polymerization process only takes place in a smaller area, and that means a smaller superresolution of the two-photon polymerization.

\section{Two-Photon Microfabrication System}

Figure 3 describes the principle of the two-photon microfabrication system with the DSE.

The real two-photon microfabrication system is shown in Figure 4. In this system, there are four subsystems.

The first subsystem is the laser subsystem. The laser subsystem contains the femtosecond laser in Figure 3. The laser is the Ti:Sapphire laser of the TIGER company, its highest output power is $1.2 \mathrm{~W}$, the laser's pulse width is more than $40 \mathrm{fs}$, and the laser's wave length range is $700-900 \mathrm{~nm}$; the highest output power of a single pulse is less than $10 \mathrm{~nJ}$. During the two-photon microfabrication process, the output power of the laser is less than $400 \mathrm{~mW}$, the center wave length is $740 \mathrm{~nm}$, the pulse width is $160 \mathrm{fs}$, and the repetitive frequency is $50 \mathrm{MHz}$.

The second subsystem is the microscope subsystem. The microscope subsystem contains DSE, resin, and confocal microscope in Figure 3. The confocal microscope is the BX51WI of the Olympus enterprise. The confocal microscope contains the infrared video, dichroic mirror, chargecoupled device (CCD), imaging lens, objective lens, focus lens, and lamp in Figure 3. The CCD is connected to a computer. The confocal microscope's former platform is replaced by three-dimension platform produced by the PI enterprise. During the two-photon microfabrication process, the bottom lighting lamp is available for the $\mathrm{S}-3$ resin. The $\mathrm{S}-3$ resin is the photonsensitive resin used in the experiments and is configured by Xi'an Jiaotong University. The DSE is placed next to the objective lens. The DSE is showed in Figure 5. The DSE makes it possible for the optimization of the spatial distribution of the laser's point spread to function (PSF) on the area of the focus.

The third subsystem is the optical subsystem. The optical subsystem contains some optical device, such as the attenuator, the beam expander, and reflection mirror in Figure 3.

The fourth subsystem is the control subsystem. The control subsystem contains the computer, monitor, threedimension platform, platform controller, and mechanical shutter in Figure 3. The computer is used to control the mechanical shutter and the three-dimension platform together to get the expose time and fabricate the threedimension microstructure.

In order to make the whole system cooperate together, the software in the computer is necessary. The main function of the software includes three-dimension track production and simulation, hardware driven, track scanning, mechanical shutter control. The software system used in our twophoton microfabrication system is programmed by Labview 


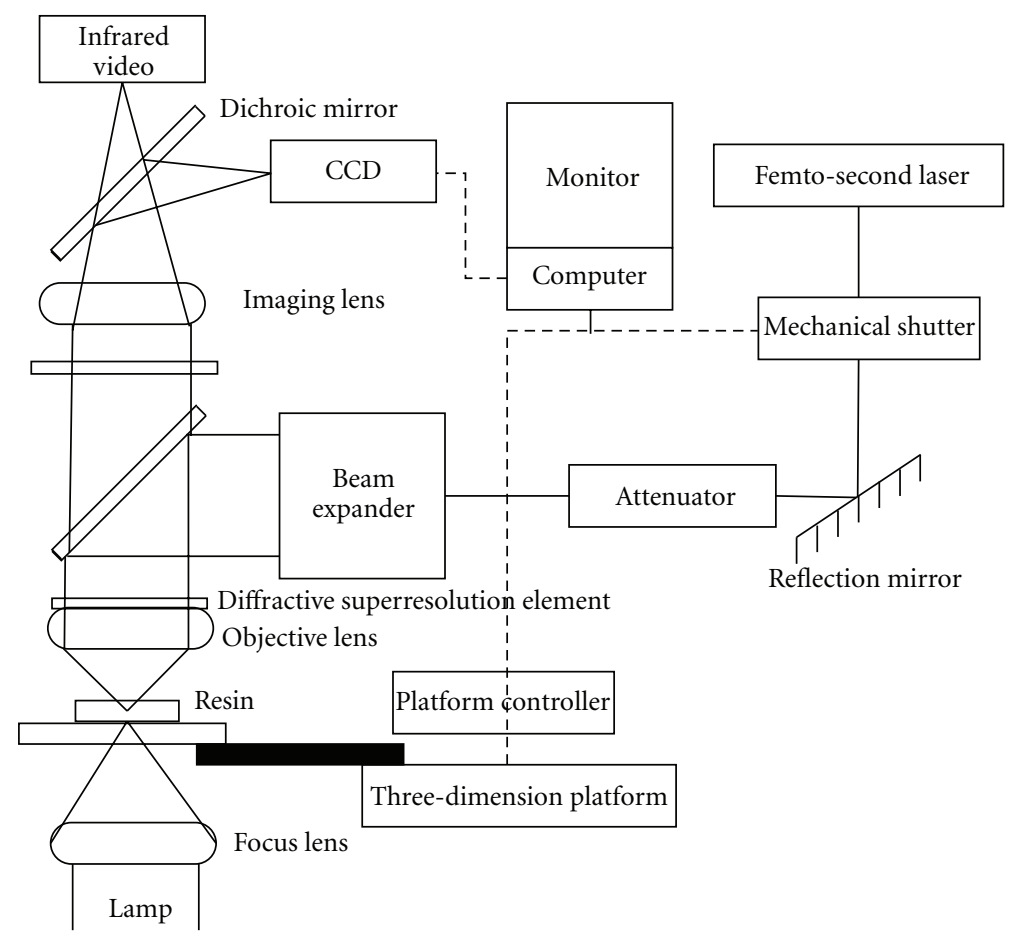

FIGURE 3: Block diagram of two-photon microfabrication system.

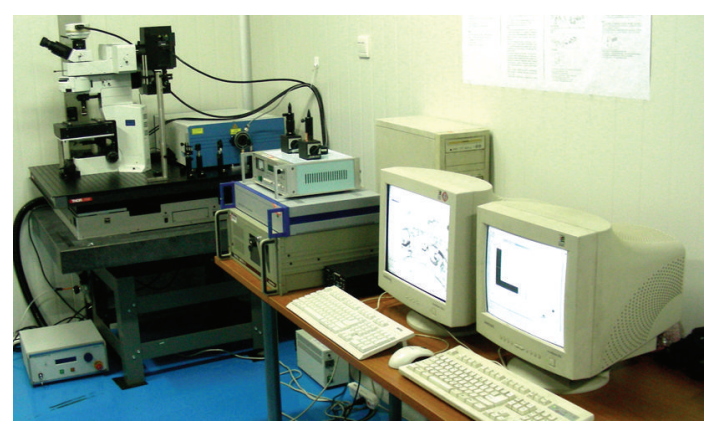

FIGURE 4: Real two-photon microfabrication system.

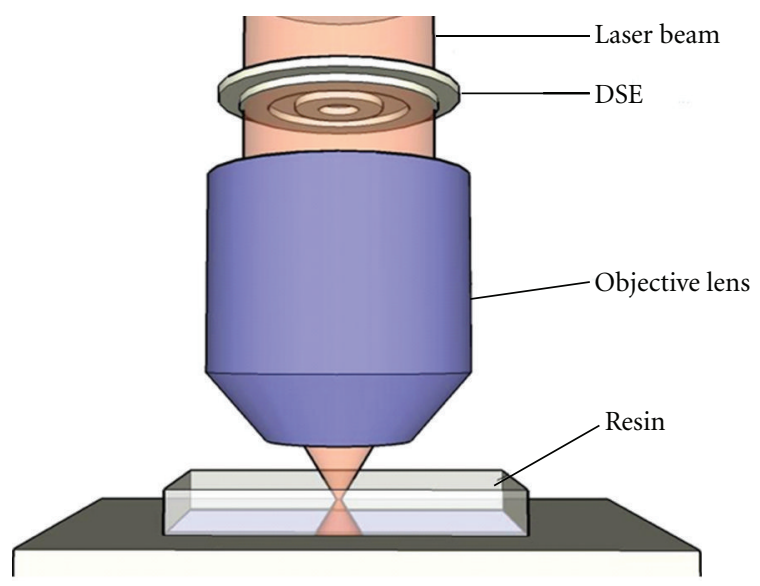

FIGURE 5: Application of the DSE in two-photon microfabrication system.

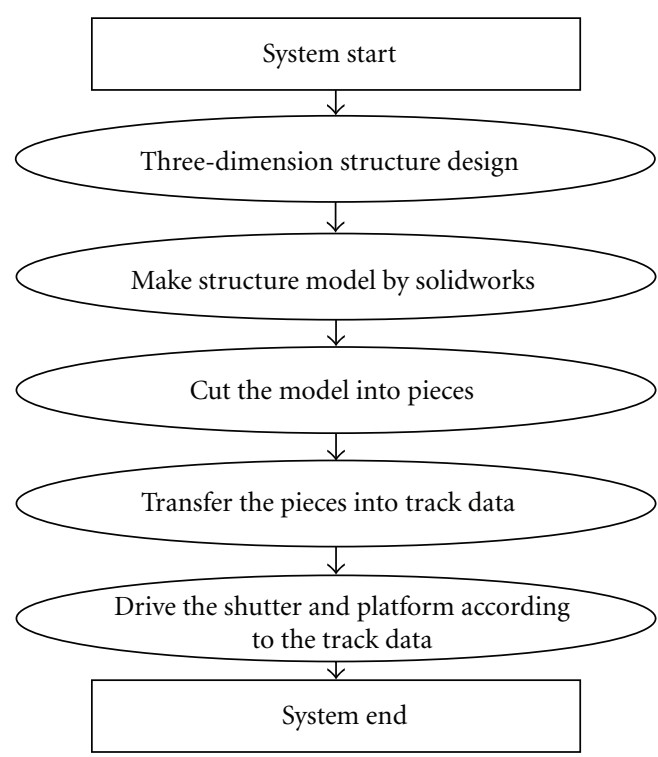

Figure 6: Software flow chart of the two-photon microfabrication system.

and $\mathrm{C}++$ language. The flow chart of the software includes seven steps and is showed in Figure 6.

The laser beam from the femtosecond laser device propagates through the shutter, the attenuator, and the expander sequentially and becomes a plane wave. Then the plane wave propagates the DSE and the objective lens continuously and focuses on the photosensitive resin. 


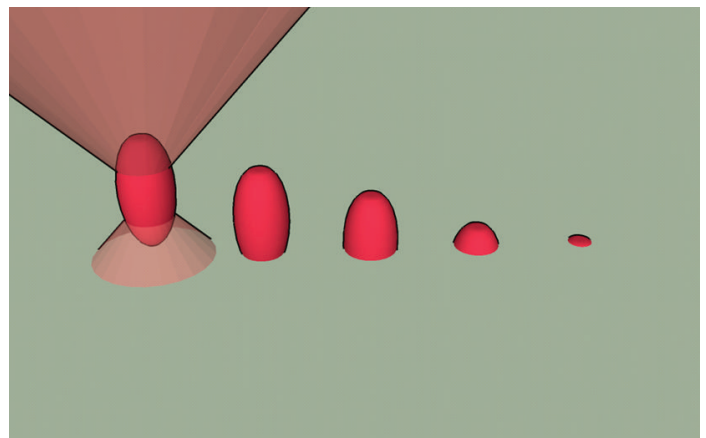

Figure 7: Simulation picture of single dots fabrication.

\section{Experiment}

4.1. Superresolution Experiments. The resolution is one of the most important characters of the two-photon microfabrication system. So the superresolution experiments are made first.

4.1.1. Single-Dot Experiment. The single-dot experiment means that the single dots are solidified in the resin under certain expose time and laser power. Figure 7 is the simulation picture of the single-dot experiment, a group of dots are fabricated on the surface of the glass base to show the resolution. In the single-dot experiment, the DSE is not used.

The real dots picture taken by optical microscope and electron microscope is shown in Figure 8.

Studying radial single-dot resolution results at different expose time and different laser power, the relation between resolution and expose time and laser power is identified and is shown in Figure 9.

It can be seen from Figure 9 that the radial resolution rises when the laser power and the expose time rise. During the dots experiment, immersion objective lens $(\mathrm{NA}=1.3)$ and $\mathrm{S}-3$ resin are used.

Dot experiment is a normal way to get the resolution of the two-photon microfabrication system. But it has a fault that the whole dot is not available in the experiment. Because the dots must be solidified on the glass base, only some part of the whole dot is shown by the microscope. So the line experiment is designed to show the resolution of the twophoton microfabrication.

4.1.2. Line Experiment without DSE. To show the spatial resolution successfully, the hanging line experiment is designed.

The hanging line experiment method is showed in Figure 10. Firstly, two erection walls are fabricated, and the distance between them is $5 \mu \mathrm{m}$. Then the lines are fabricated between them with the two-photon microfabrication system. And finally the superresolution of the two-photon microfabrication is showed in the lines by the electron microscope.

Figure 11 shows the superresolution of the two-photon microfabrication system, in which the radial superresolution is showed by the platform of the hanging line in Figure 11(a),

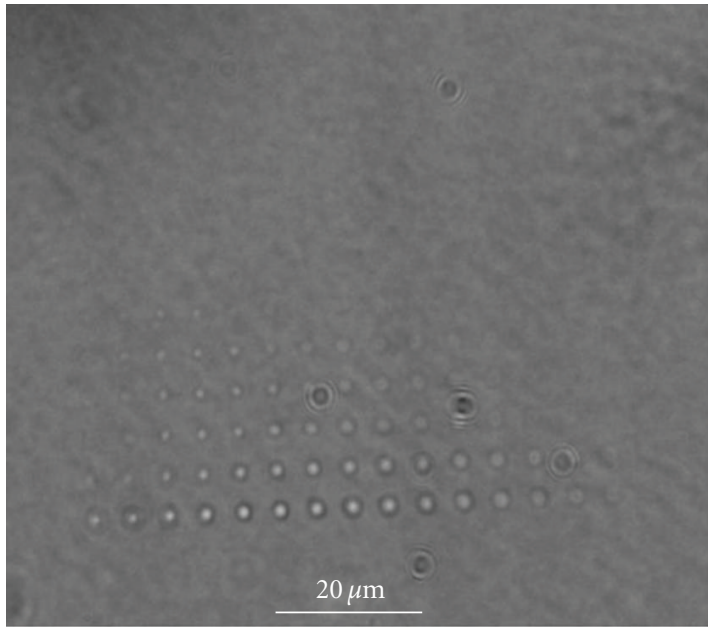

(a)

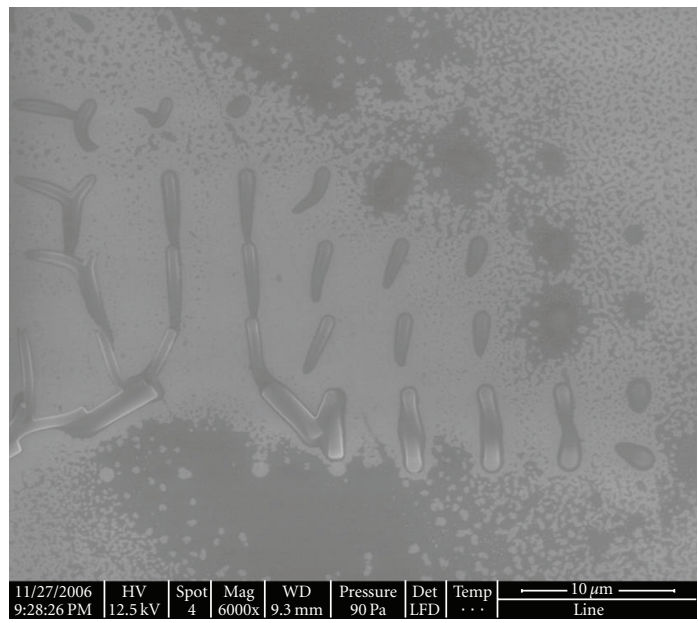

(b)

FIGURE 8: Dot picture under (a) optical microscope (b) electron microscope.

and the axial superresolution is showed by the side view of the hanging line in Figure 11(b).

Before using the DSE, the minimum superresolution of the line in the experiment is showed in Figure 12. In the experiment, the objective lens is immersion lens $(\mathrm{NA}=1.3$, $n=1.516$ ), and the center wavelength of the femtosecond laser is $750 \mathrm{~nm}$.

It can be seen from Figure 12 that the minimum superresolution of the line before using DSE is more than $100 \mathrm{~nm}$.

4.1.3. Line Experiment with DSE. Keeping the same experiment conditions as the line experiment without DSE, the line experiment with DSE is done. The DSE is placed in the twophoton microfabrication system showed in the Figure 5. Because the adjusting amplitude DSE can cause some energy loss, the laser output power is adjusted to 60-90 mW. Finally, the minimum superresolution with the DSE is showed in Figure 13. 


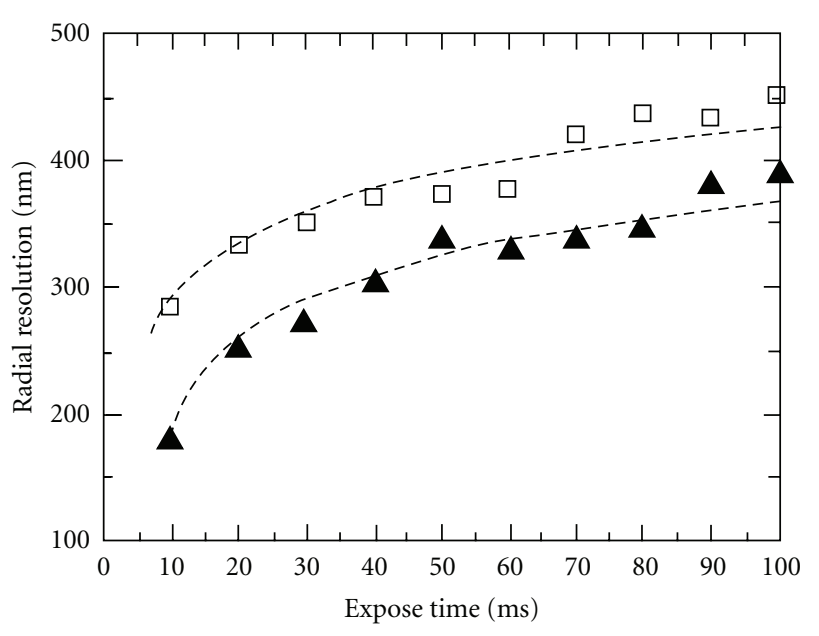

A $15 \mathrm{~mW}$

$\square \quad 45 \mathrm{~mW}$

Figure 9: Radial resolution at different expose time and different laser power.

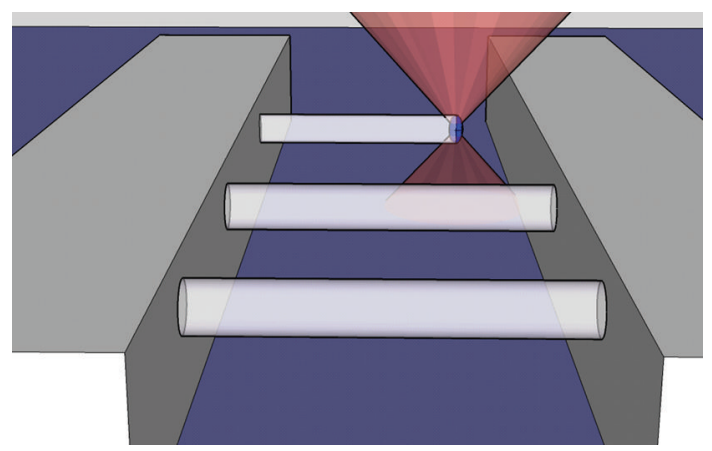

FIgURE 10: Hanging line experiment of the two-photon microfabrication.

It can be seen from the experiment result showed in Figure 13 that the minimum superresolution of the line after using DSE is less than $50 \mathrm{~nm}$. And comparing the results showed in Figures 12 and 13, it can be seen that the minimum superresolution of the two-photon microfabrication system with DSE is better than the minimum result without DSE.

4.2. Microfabrication Experiments. Some experiments are done in the lab.

4.2.1. Single Three-Dimension Model. Two-photon microfabrication system can make three-dimension model. Figure 14 is a Chinese seal-the official emblem of the 2008 Summer Olympics in Beijing. Figure 14(a) is the track of the Chinese seal in the computer; Figure 14(b) is the fabrication result of the Chinese seal by the two-photon microfabrication system.

Figure 15 is the Chinese map and Figure 15(a) is the track of the Chinese map in the computer, Figure 15(b) is

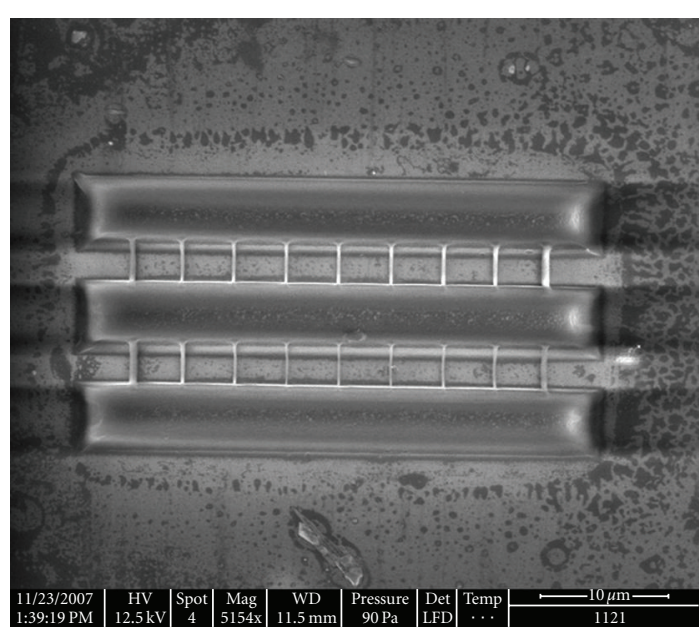

(a) Planform

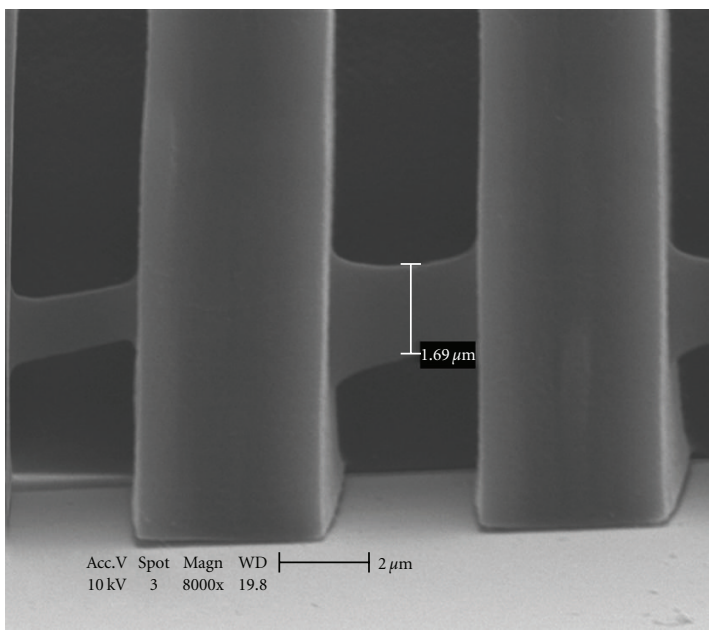

(b) Side view

Figure 11: Hanging line experiment result of the two-photon microfabrication.

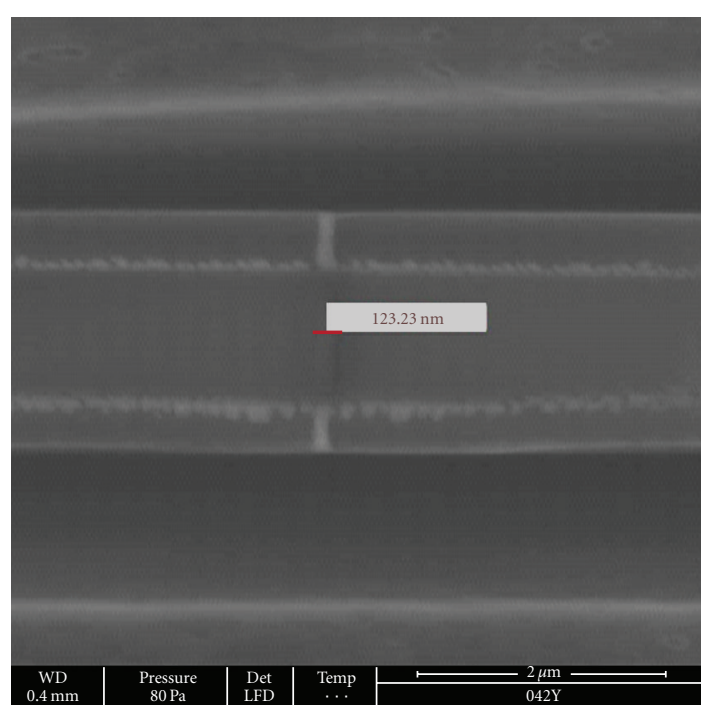

FIgURE 12: Minimum superresolution line without DSE. 


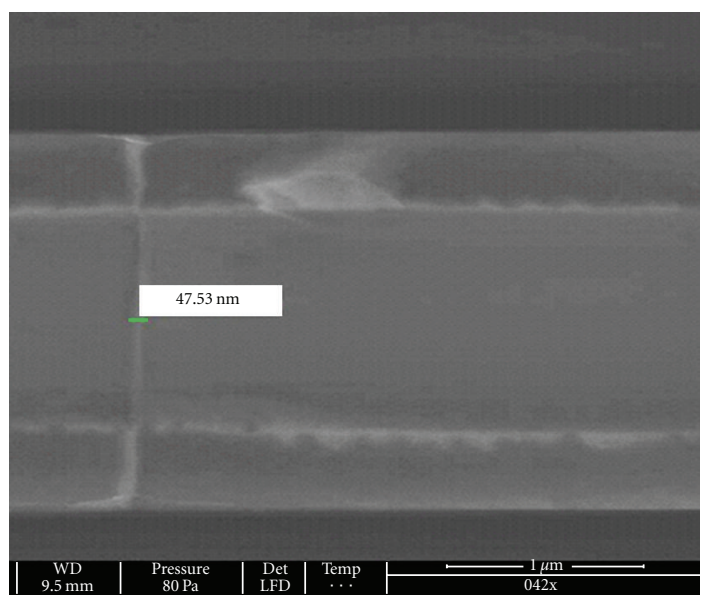

FIgure 13: Minimum superresolution line with DSE.

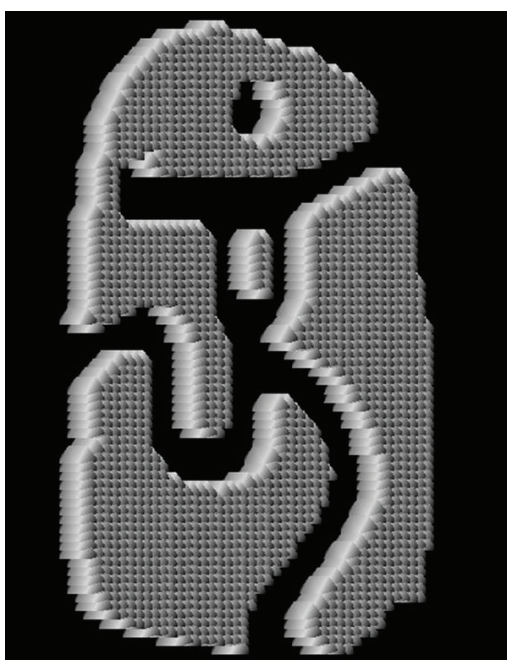

(a) Track

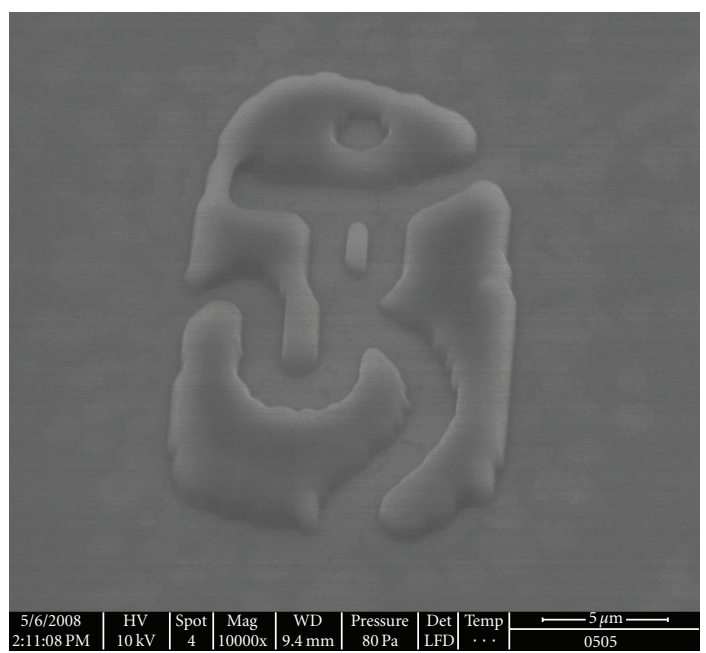

(b) Microfabrication result

Figure 14: Chinese seal.

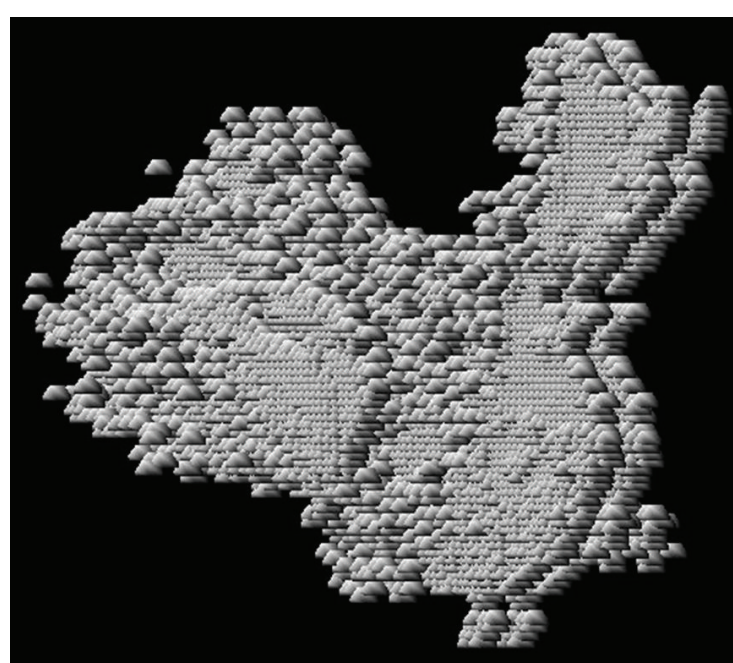

(a) Track

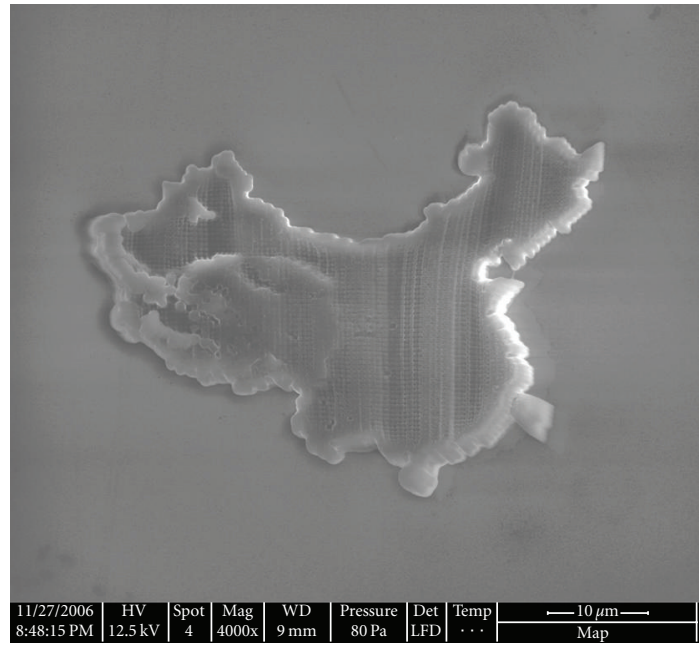

(b) Microfabrication result

Figure 15: Chinese map.

the fabrication result of the Chinese map by the two-photon microfabrication system.

Figure 16 is a three-dimension brand. Figure 16(a) is the track of the brand in the computer, and Figure 16(b) is the fabrication result of the three-dimension brand by the twophoton microfabrication system.

4.2.2. Assembled Three-Dimension Model. Two-photon microfabrication system cannot only make single threedimension models but some assembled model system. Figure 17 is a gear and a bracket assembled together. This set of three-dimensional models is fabricated together at the same time by the two-photon microfabrication system, and the gear can move freely in the bracket. During the fabrication process, the $\mathrm{S}-3$ resin and objective lens $(\mathrm{NA}=$ 0.8 ) are used.

Figure 18 is a leaf and a bracket assembled together. This set of three-dimensional model systems is fabricated together at the same time by the two-photon microfabrication system. 


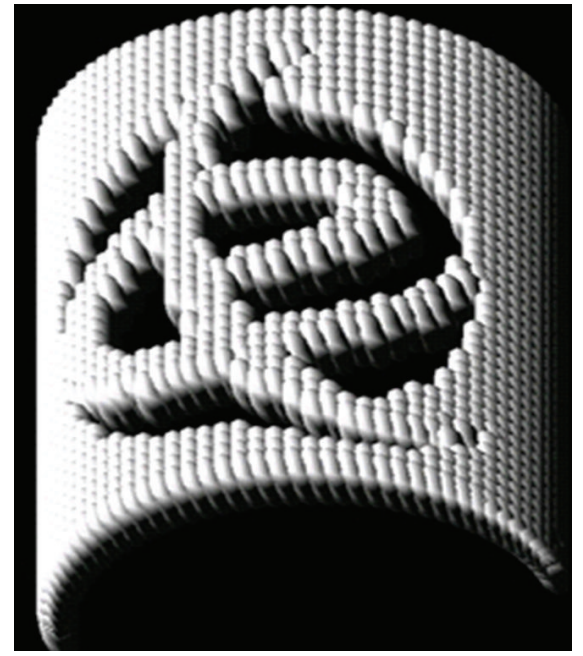

(a) Track

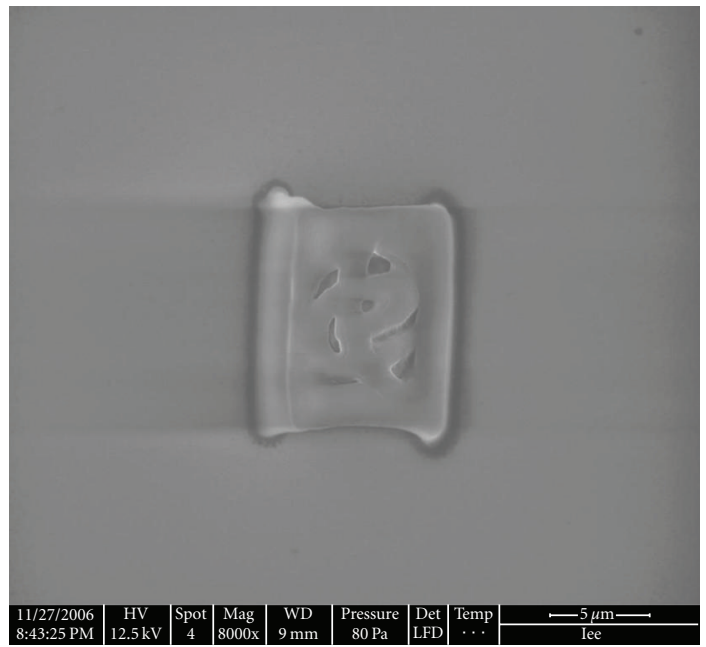

(b) Microfabrication result

Figure 16: Three-dimension brand model.

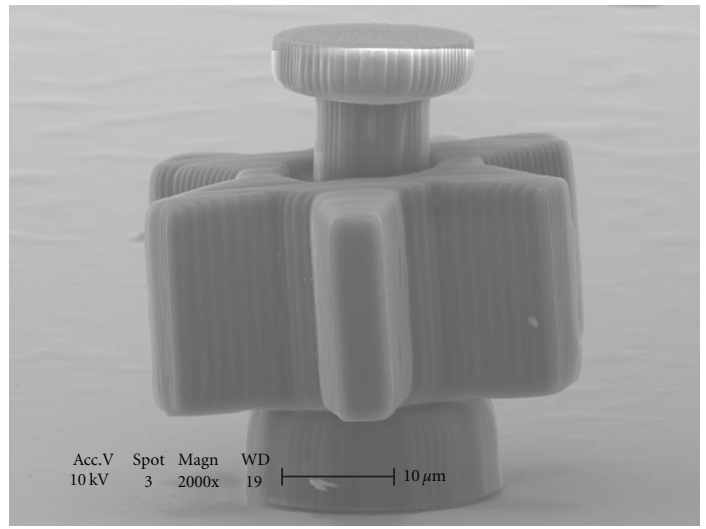

FIGURE 17: Gear and bracket.

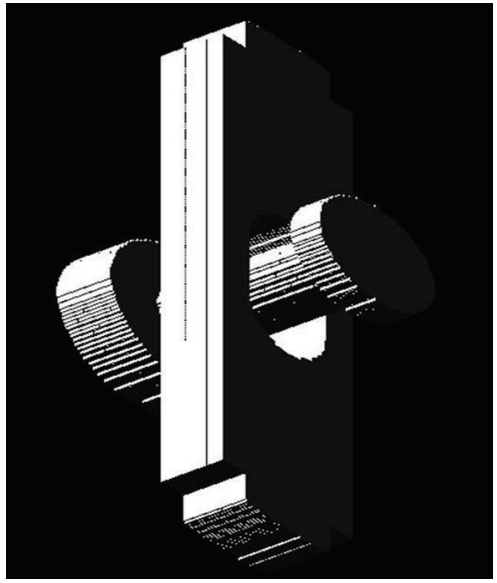

(a) Track

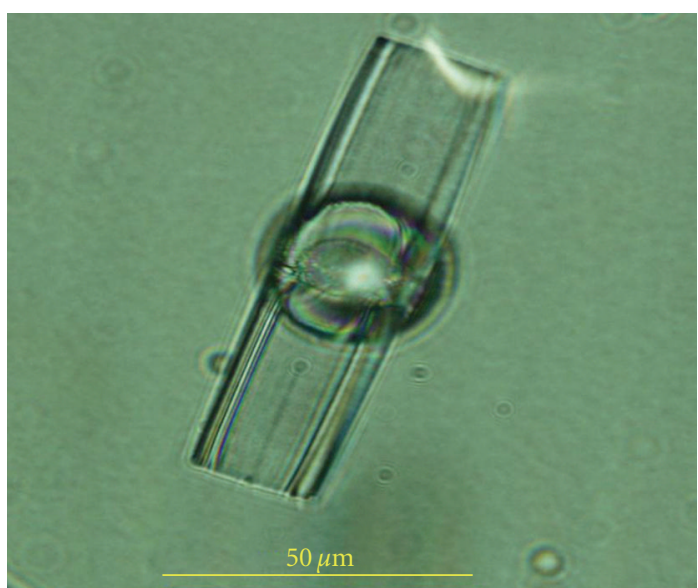

(b) Leaf and bracket

Figure 18: Leaf and bracket.

During the fabrication process, the S-3 resin and objective lens $(\mathrm{NA}=0.8)$ are used. The length of the leaf is $80 \mu \mathrm{m}$, the distance between the leaf and the bracket is $3 \mu \mathrm{m}$, and the driven experiment shows that the leaf can move in the bracket.

\section{Summary}

During the whole experimentations period, we experienced difficulties including the resin character, the position of the lamp and DSE, the software programming, and the control of the shutter and the platform, but the experimentation shows that the two-photon microfabrication system is a unique fabrication way. The superresolution can be improved by a properly designed DSE, laser power, and expose time. And experiment results illustrate the flexibility of this technology. Finally, assembled 3D microstructures system could be fabricated through this technique. Therefore, this process provides some references to develop new conceptive nano/ microdevices in many areas. 


\section{Acknowledgments}

This work was supported by the Project 973 (no. 2003CB716204) and the National Science Foundation (no. 50875015) in China.

\section{References}

[1] P. Wei, O. F. Tan, Y. Zhu, and G. H. Duan, "Axial superresolution of two-photon microfabrication," Applied Optics, vol. 46, no. 18, pp. 3694-3699, 2007.

[2] X. Q. Wei, The resolution enhancement of the two-photon microfabrication and its application, M.S. dissertation, Chinese Academy of Science, 2008.

[3] D. Tan, Y. Li, F. Qi et al., "Reduction in feature size of twophoton polymerization using SCR500," Applied Physics Letters, vol. 90, no. 7, Article ID 071106, 2007.

[4] L. Li, R. R. Gattass, E. Gershgoren, H. Hwang, and J. T. Fourkas, "Achieving 1/20 resolution by one-color initiation and deactivation of polymerization," Science, vol. 324, no. 5929, pp. 910-913, 2009.

[5] S. Kawata, H. B. Sun, T. Tanaka, and K. Takada, "Finer features for functional microdevices," Nature, vol. 412, no. 6848, pp. 697-698, 2001.

[6] B. H. Cumpston, S. P. Ananthavel, S. Barlow et al., "Twophoton polymerization initiators for three-dimensional optical data storage and microfabrication," Nature, vol. 398, no. 6722, pp. 51-54, 1999.

[7] J. Serbin, A. Egbert, A. Ostendorf et al., "Femtosecond laserinduced two-photon polymerization of inorganic-organic hybrid materials for applications in photonics," Optics Letters, vol. 28, no. 5, pp. 301-303, 2003.

[8] S. Juodkazis, V. Mizeikis, K. K. Seet, M. Miwa, and H. Misawa, "Two-photon lithography of nanorods in SU-8 photoresist," Nanotechnology, vol. 16, no. 6, pp. 846-849, 2005.

[9] W. Haske, V. W. Chen, J. M. Hales et al., "65 nm feature sizes using visible wavelength 3-D multiphoton lithography," Optics Express, vol. 15, no. 6, pp. 3426-3436, 2007.

[10] D. Stoppa, D. Mosconi, L. Pancheri, and L. Gonzo, "Singlephoton avalanche diode CMOS sensor for time-resolved fluorescence measurements," IEEE Sensors Journal, vol. 9, no. 9, pp. 1084-1090, 2009.

[11] Z. W. Jiang, D. J. Yuan, A. D. Zhu et al., "The development of the two-photon three-dimension microfabrication technology and the experiment system," Optics and Precision Engineering, vol. 3, pp. 234-238, 2003.

[12] Z. W. Jiang, The analysis of the femtosecond laser twophoton three dimension microfabrication, Ph.D. dissertation, University of Science and Technology of China, 2004.

[13] Z. W. Jiang, D. J. Yuan, R. Guo et al., "Analysis on the resolution of two-photon three-dimension microfabrication," Microfabrication Technology, vol. 2, pp. 30-36, 2004.

[14] Z. W. Han, Macromolecule Science Tutorial, Trans Huadong Science and Technology University Publications, Shanghai, China, 2001.

[15] H. Liu, Y. Yan, Q. Tan, and G. Jin, “Theories for the design of diffractive superresolution elements and limits of optical superresolution," Journal of the Optical Society of America A, vol. 19, no. 11, pp. 2185-2193, 2002.

[16] H. Liu, Y. Yan, D. Yi, and G. Jin, "Theories for the design of a hybrid refractive-diffractive superresolution lens with high numerical aperture," Journal of the Optical Society of America A, vol. 20, no. 5, pp. 913-924, 2003.
[17] H. T. Liu, Investigations of design methods of diffractive optical elements to implement optical superresolution, Ph.D. dissertation, Tsinghua University China, 2004.

[18] T. G. Jabbour, M. Petrovich, and S. M. Kuebler, "Design of axially super-resolving phase filters using the method of generalized projections," Optics Communications, vol. 281, no. 8, pp. 2002-2011, 2008.

[19] T. G. Jabbour and S. M. Kuebler, "Particle-swarm optimization of axially superresolving binary-phase diffractive optical elements," Optics Letters, vol. 33, no. 13, pp. 1533-1535, 2008.

[20] T. G. Jabbour and S. M. Kuebler, "Axial field shaping under high-numerical-aperture focusing," Optics Letters, vol. 32, no. 5, pp. 527-529, 2007. 

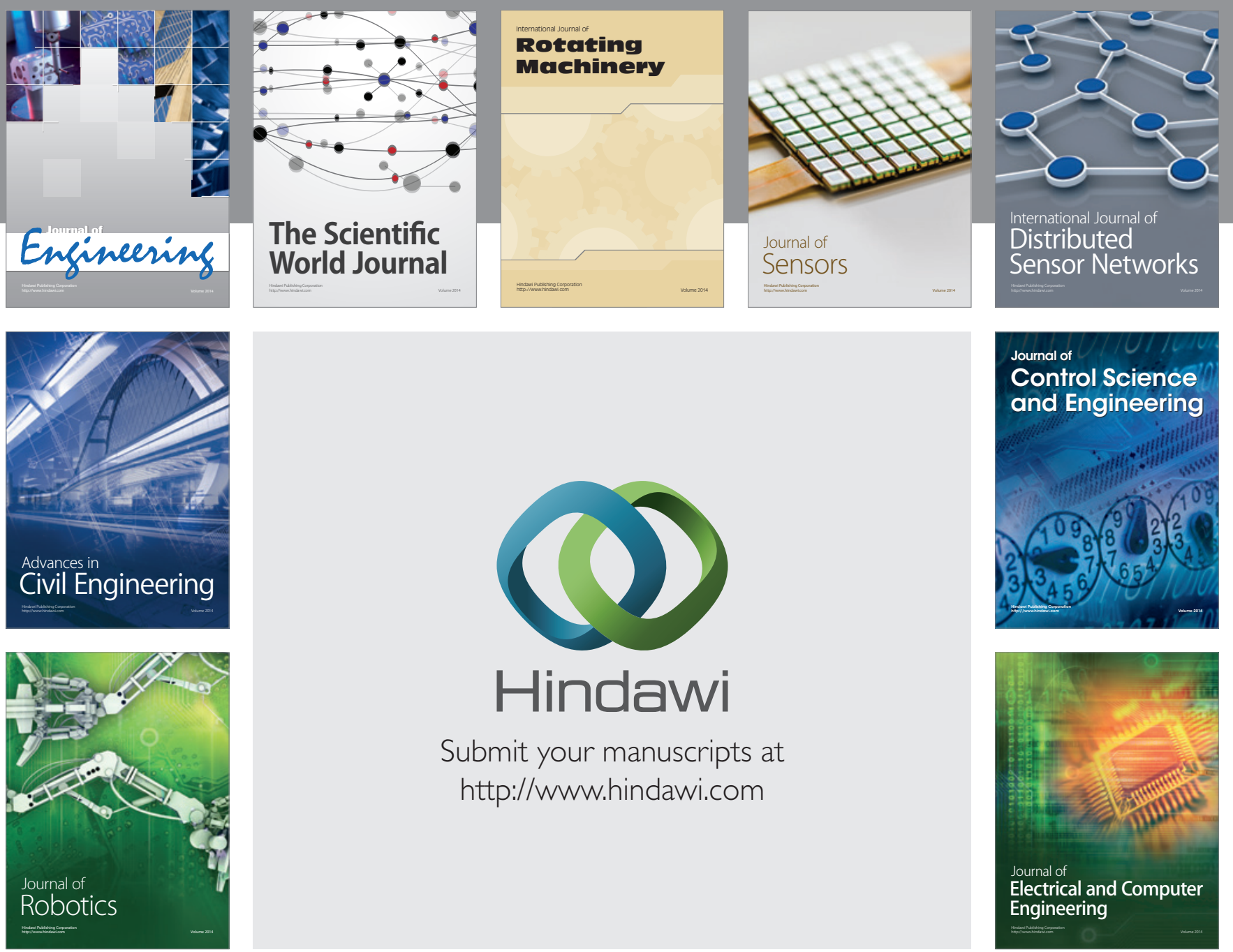

Submit your manuscripts at

http://www.hindawi.com
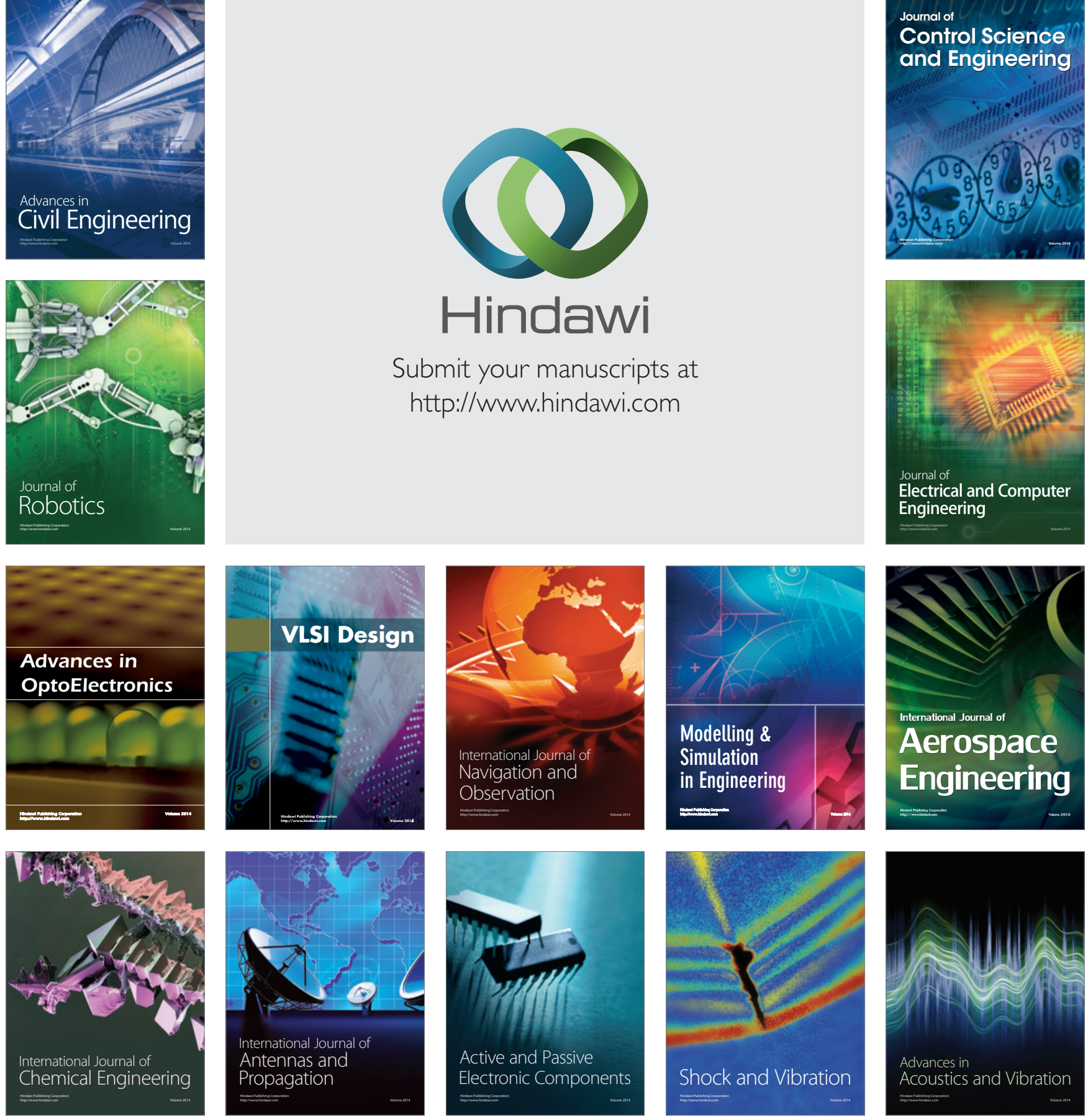